\title{
DISPLACEMENT SENSING BY FIELD EMISSION WITH NANOMETER RESOLUTION
}

\author{
A.J. le Fèbre, M. Siekman, L. Abelmann and J.C. Lodder \\ MESA+ Institute for Nanotechnology, University of Twente, Enschede, NETHERLANDS \\ (Tel : +31-53-489-4673; E-mail: a.j.lefebre@utwente.nl)
}

\begin{abstract}
Field emission is used as a displacement sensing method, exploiting the exponential relation between field emission current and electrode gap. Atomic force microscopy (AFM) probes have been used as field emission source to measure I/V characteristics which were found to correspond well to theory. The field emission sensor was operated in a more linear regime by using feedback on the position of the probe in order to maintain a constant current. The sensitivity of the sensor for displacement was found to be $0.26 \mathrm{~V} / \mathrm{nm}$ at a range of $\sim 100 \mathrm{~nm}$. From the experimental data, typical parameters for the Fowler-Nordheim equation were deduced and used to model the sensor performance. The measurements confirm that field emission can be applied to sense the distance between a probe tip and sample with $<20 \mathrm{~nm}$ resolution.
\end{abstract}

Keywords: Field emission, sensor, displacement sensing, probe recording

\section{INTRODUCTION}

In this paper, we present measurement results of displacement sensing using field emission and provide more insight in the environmental (vacuum) conditions and electronics needed for stable operation of field emission in MEMS/NEMS sensor applications.

Sensors based on the field emission effect can be used to detect displacements up to hundreds of nanometers with nanometer resolution. This sensitivity arises from the exponential dependence of the field emission current on the electrode spacing, normally between a tip field emission source and a counter-electrode. Various types of sensors based on field emission have already been developed for MEMS, e.g. for pressure [1], acceleration [2] or RF applications [3]. Field emission has significant advantages over other methods used for displacement sensing, i.e. the measured current signal is largely temperature independent, the critical sensing area can be scaled down to the nanometer range and easily integrated, without sacrificing sensitivity or signal-to-noise ratio. However, the conditions that influence the stability of the field emission process are often not well controlled.

Our aim is to apply this technique in a probe storage system, where thousands of probes are individually positioned at several nanometers above a magnetic recording medium [4] .

\section{THEORY}

Fowler-Nordheim theory describes the field emission process in terms of a tunnelling current through a potential barrier from a metal surface into vacuum [5]. This current is given by

$$
I=\lambda A a \phi^{-1} E^{2} \exp \left\{-\mu b \phi^{3 / 2} / E\right\}
$$

with $A$ the area of emission, $\phi$ the local work function of the emitting surface, $E$ the applied electric field, $a$ and $b$ constants and $\lambda$ and $\mu$ factors to correct for mathematical approximations and physical assumptions on the potential barrier shape [6].

The electric field $E$ between a tip and flat electrode can be given by

$$
E=\gamma V / d
$$

where $V$ is the voltage and $d$ the spacing between the electrodes. The 'field-enhancement-factor' $\gamma$ relates the electric field to the electrode geometry [7]. The following simple model is used to describe the field enhancement at the apex of a sphere with radius $r$ at a distance $d$ from a flat counter-electrode:

$$
\gamma=k(r+d) /(k r+d)
$$

with $k$ a factor describing the tip sharpness. This model gives the distance dependence of the field enhancement factor: for small distance, the electrode configuration approaches a parallel plate 
configuration $(\gamma \approx k r / k r=1)$; for large distance, the field enhancement factor saturates to a maximum value $(\gamma \approx k)$. Sharper tips have a larger $k$ and thus a larger field enhancement.

\section{EXPERIMENTAL}

An UHV scanning probe microscope was used to investigate the characteristics of field emission sensing. Details on the measurement setup have been described previously [4]. The microscope scanner was used to approach individual field emitter probes to a counter-electrode of $\sim 100 \mathrm{~nm}$ TiW sputtered on a silicon wafer sample. Probe displacements were limited to $1 \mu \mathrm{m}$ by the range of the piezo actuators.

As field emitters we used AFM probes, which consist of a sharp tip on a cantilever. Highly doped $(<0.01 \mathrm{Ohm} \cdot \mathrm{cm})$ single crystal silicon probes (Nanosensors PPP-NCLR) were used, with a high force constant $(48 \mathrm{~N} / \mathrm{m})$ to prevent pull-in of the cantilever. The tip height is $10-15 \mu \mathrm{m}$ with a tip radius of typically $<7 \mathrm{~nm}$.

Instabilities in the field emission current are known to arise from atomic changes of the field emitter and sample surfaces [8]. Also deposition can take place, induced by field emitted electrons that dissociate adsorbed hydrocarbons on the surface [9]. To prevent field emission induced deposition and improve the stability of the field emission current, emitters and counter-electrode were annealed and the UHV system frequently baked and operated at $5 \cdot 10^{-10}$ mbar.

\section{RESULTS AND DISCUSSION}

\subsection{Field emission at small distances}

Measurements of the distance dependence of the field emission I/V characteristics are shown in Fig. 1a. Here the sensor probe was brought into contact with the sample. Next the probe was retracted to increase the probe-sample distance to $50 \mathrm{~nm}$. A positive bias sweep was applied to the sample to extract current from the probe tip. This measurement was repeated for increasing probesample distances from $50 \mathrm{~nm}$ to $950 \mathrm{~nm}$.

In Fig. 1b, the I/V data is plot on a semilogarithmic scale, resulting in so-called FowlerNordheim plots. The straight lines clearly indicate that the current acquired is due to the field emission effect. By using a special fitting procedure described in [6], we obtain the typical parameters for the Fowler-Nordheim equation with reasonable reliability, i.e. the emission area and the field enhancement factor. If we assume the work function to be that of silicon, $\phi=4.5 \mathrm{eV}$, we obtain an average value for the emission area of $A=3.5 \cdot 10^{-17} \mathrm{~m}^{2}$. The field enhancement factor is determined from the line slopes (Fig. 1b) and is clearly varying with the distance between probe and sample at which each curve is taken.
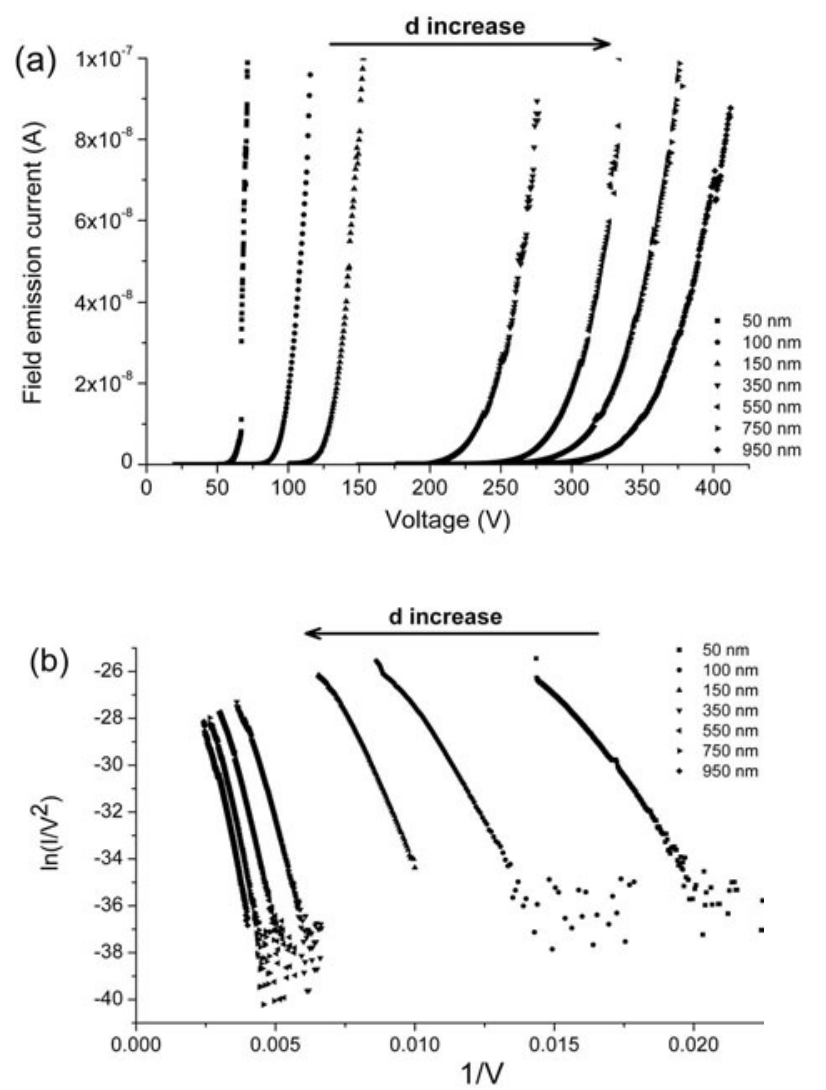

Fig. 1. Distance dependence of field emission in (a) $I / V$ characteristics and (b) corresponding Fowler-Nordheim plots, measured for gaps from $50 \mathrm{~nm}$ to $950 \mathrm{~nm}$ between standard AFM probe and silicon sample coated with $100 \mathrm{~nm} \mathrm{TiW}$.

In Fig. 2 the field enhancement factors are plotted as function of the tip-sample distance. The simple model of Eq. (3) is used to fit the data points, by choosing values for $r$ and $k$. An optimal fit was obtained for $r=40 \mathrm{~nm}$, which could be the tip radius of a slightly damaged AFM probe, and $k=25$, which determines the maximum field enhancement at larger distance. 


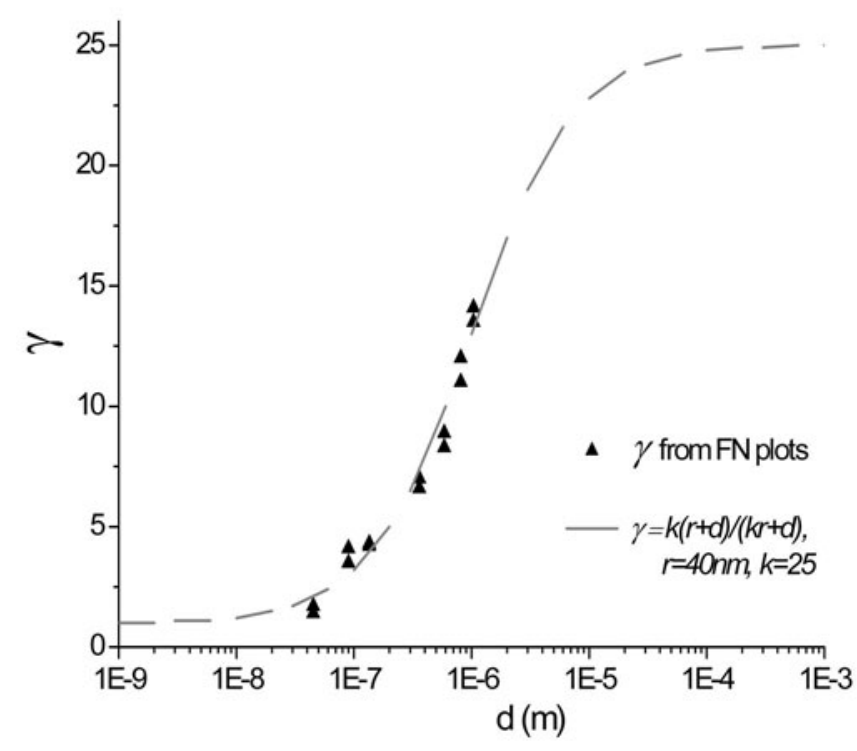

Fig. 2. Field enhancement factors determined by fitting the Fowler-Nordheim plots (Fig. 1b), plotted as function of electrode distance. Line is fitting result of Eq. (3) with $r=40 \mathrm{~nm}$ and $k=25$.

\subsection{Field emission to control tip-sample distance}

The exponential nature of the field emission current gives rise to unstable behaviour, most likely caused by the atomic changes of the field emitter and sample surfaces. By keeping the field emission current constant using position feedback, the sensor can be operated in a more linear regime and used for displacement sensing [10].

In Fig. 3 the relationship between displacement and voltage was measured by using feedback on the positioning actuator of the probe in order to maintain a constant current of $3 \mathrm{nA}$. By increasing the applied voltage from 5 to $40 \mathrm{~V}$, the probe is retracted from the sample to keep the current constant. Below $5 \mathrm{~V}$, there is a transition to the 'normal' tunnelling regime. The accuracy of positioning during a single sweep was found to be less than $1 \mathrm{~nm}$. However, instabilities in the field emission current cause larger changes in the probe position and limit the positioning repeatability to $<20 \mathrm{~nm}$ when repeating the experiment 10 times.

The relation between displacement and voltage is fixed by the constant electric field at which field emission takes place. The sensitivity is therefore determined by the field enhancement factor, thus by the probe tip shape. This explains the nonlinearity of the curves: with variation of the probe position, also the field enhancement factor changes. At low voltage, the probe is close to the sample and the field enhancement factor is $\sim 1$. With increasing voltage the probe is further retracted and the field enhancement increases. When the field enhancement saturates to its maximum value, the displacement sensitivity will show a linear relation.

The measurement data was fitted using the model from Eq. (3), the Fowler-Nordheim equation (1) and the field emission parameters from Fig. 1. The best fit was obtained by using $r=5 \mathrm{~nm}$ and $k=25$. Note that this measurement is on a tip different from Fig. 2, with a tip radius typical for an undamaged AFM probe. Due to this smaller $r$ the field enhancement reaches its maximum value at smaller distance. The $k$ factor determines the slope of the curve at larger voltages and was chosen $k=25$ in order to give the best fit to the data points. This value corresponds well to that used in the fit of Fig. 2. Using these parameters, the displacement curve becomes linear for gaps $>100 \mathrm{~nm}$, with a displacement sensitivity of $0.26 \mathrm{~V} / \mathrm{nm}$.

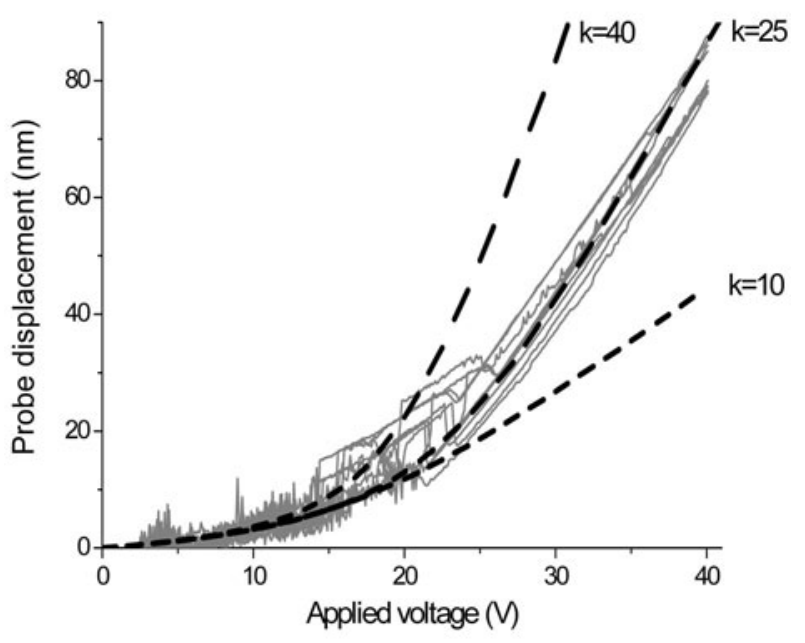

Fig. 3. Displacement measured as function of applied bias voltage (constant current $3 \mathrm{nA}$ ). Fits determined by model from Eq. (3) using $r=5 \mathrm{~nm}$.

\subsection{Influence of cantilever deflection}

We use AFM probes with a cantilever, since the cantilever deflection can ultimately be used to sense magnetic forces. Due to electrostatic interaction between the AFM probe and the sample, the cantilever also deflects during the measurements presented in Fig. 3. 
A different measurement setup and AFM probe were used to investigate the influence of cantilever deflection during a voltage sweep, see Fig. 4. In this graph, the upper curve is the position of the probe, determined from the feedback signal to the position actuator. The bottom curve gives the cantilever deflection, which saturates to a maximum value of $\sim 20 \mathrm{~nm}$ for higher voltage. This confirms that the high force constant $(48 \mathrm{~N} / \mathrm{m})$ of the cantilever is sufficient to limit the deflection. The resulting effective distance between the probe tip and the sample is the sum of the two curves and is also included in the figure. This curve is more linear compared to the curve given in Fig. 3, which is not completely understood. However, this measurement clearly shows that the displacement due to the field emission effect is dominant over the cantilever deflection.

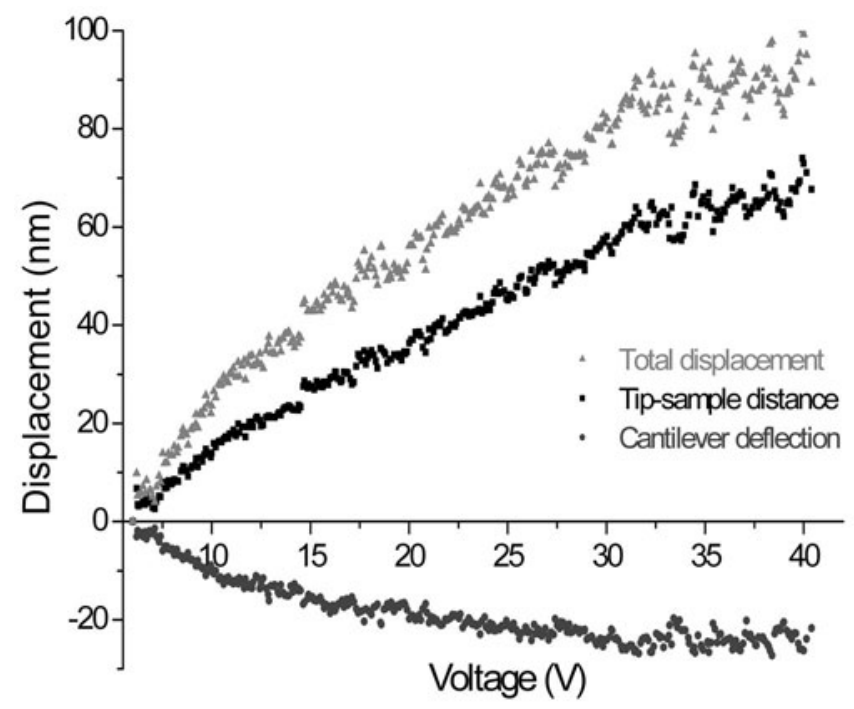

Fig. 4. Displacement, cantilever deflection and tip-sample distance as function of applied voltage during feedback operation (constant current $3 \mathrm{nA}$ )

\section{CONCLUSIONS}

Field emission is shown from AFM probes at varying distances from a counter-electrode sample. By operating the probes in constant current mode, it is possible to control displacements with nanometer sensitivity. To improve stability, clean surfaces and UHV conditions were used, however the reproducibility is still limited to $<20 \mathrm{~nm}$. For linear operation, the probe should be used in a range with constant field enhancement. Therefore the relationship between tip shape and field enhancement needs better understanding. Since field emission displacement sensing can be used to position individual probes with nanometer resolution, the method may result in an integrated method for probe positioning in a MEMS probe storage system. However, for practical applications, the field emission stability should be sufficient also in poor vacuum conditions, which may be achieved by using other field emitter materials.

\section{ACKNOWLEDGEMENTS}

This work is part of the STW program 'Micro Scanning Probe Array Memory'. The authors thank T. Bolhuis for technical assistance and R. Luttge for sample preparation.

\section{REFERENCES}

[1] H.H. Busta, J.E. Pogemiller, B.J. Zimmerman, J. Micromech. Microeng. 3, 49, (1993).

[2] A. Hariz, H.G. Kim, M.R. Haskard and I.J. Chung, J. Micromech. Microeng. 5, 282, (1995).

[3] K. Yamashita, W. Sun, K. Kakushima, H.Fujita, H. Toshiyoshi, J. Vac. Sci. Technol. B 24(2), 927, (2006).

[4] A.J. le Febre, R. Luttge, L. Abelmann, J.C. Lodder, ICNT2006. Accepted for publication in Journal of Physics: Conference Series (JPCS).

[5] H. Fowler, L.W. Nordheim, Proc. Roy. Soc. London A119 173, (1928).

[6] R.G. Forbes, Ultramicroscopy 79, 11, (1999).

[7] R.G. Forbes, C.J. Edgcombe, U. Valdré, Ultramicroscopy 95, 57, (2003).

[8] T. T.Tsong, IEEE Trans. Electron Devices 38, 2317, (1991).

[9] M. A. McCord, D. P. Kern, T. H. P. Chang, J. Vac. Sci. Technol. B 6, 1877, (1988).

[10] R. Young, J. Ward, F. Scire, Rev. Sci. Instrum. 43, 999, (1972). 